\title{
Computational and experimental diagnostics of the shear properties of greases
}

\author{
Aleksandr Dykha, ${ }^{1, *}$,Serhii Matiukh ${ }^{1}$, Marcin Łukasiewicz ${ }^{2}$, Natalia Dluhunovych ${ }^{1}$ \\ ${ }^{1}$ Khmelnitskyi National University, 29016, Instytutska 11, Khmelnitskyi, Ukraine \\ ${ }^{2}$ Bydgoszcz University of Science and Technology, Al. prof. S. Kaliskiego 7, 85-796 Bydgoszcz, \\ Poland
}

\begin{abstract}
The study of the properties of consistent and liquid oils is currently receiving much attention in tribology. This is because lubrication is one of the most effective ways to improve machine durability. At the same time are practically no mathematical descriptions of the mechanisms of grease lubrication, which is necessary for predicting and calculating the wear of machines. When studying the wear of surfaces under boundary lubrication conditions, it is necessary to know the stiffness characteristics of a thin layer of lubricant under normal and tangential stresses. In this paper, a method is proposed for determining the function of the contact tangential characteristics of a thin oil layer between solid deformable surfaces. A thin layer of grease is placed between two hard discs. By measuring the angle of rotation and the moment on the second disc, the dependence of the moment on the angle of rotation is determined. The contact mechanics of torsion of a thin circular lubricant layer has been developed and relations for determining the parameters of the shear diagram of a thin lubricant layer have been obtained. According to the developed method, the Fiol-3 grease was tested and the parameters of the shear diagram were determined.
\end{abstract}

\section{Introduction}

Increasing the durability of machines is achieved not only by the use of new materials, coatings, but by the correct choice of lubricant. For the selection of oils, the most reliable tests are in real operation, but they are expensive. Therefore, laboratory tests are also used. Laboratory tests of lubricants are carried out to determine the physical and chemical properties. Liquid and grease lubricants, separating surfaces with a thin layer, are most commonly used to reduce wear. For testing liquid lubricants, a four-ball test scheme is used. The use of test results is qualitative in nature if there are no solutions to the contact problem with wear for a pair of balls. The authors of this study proposed a method of testing for wear with lubrication according to a four-ball scheme with the determination of the parameters of wear models [1]. The effectiveness of a grease is determined by how long

\footnotetext{
*Corresponding author: tribosenator@gmail.com
} 
it remains on the surface. The effectiveness of greases is determined by the shear strength of the grease. After the destruction of the framework, the lubricant begins to deform like a liquid. The resistance to flow of a grease is characterized by its viscosity. Viscosity is a property of a grease that depends on the rate of deformation. Typically, viscosity is determined at one fixed strain rate. Many friction units of machines operate under conditions of boundary lubrication of the contacting surfaces. Experimental determination of the shear stress in a loaded lubricated contact is important for analyzing the operation of friction units. In this paper, a method is proposed for determining the function of the contact tangential characteristics of a thin oil layer between solid deformable surfaces.

The idea of the method is as follows. A thin layer of grease is placed between two hard discs. The drive disc starts to turn slowly and passes through the lubricant to the other disc. By measuring the angle of rotation and the moment on the second disc, the dependence of the moment on the angle of rotation is determined. This dependence contains information about the distribution of tangential forces in the lubricant between the discs. Using a mathematical model of film deformations, it is possible to obtain a film shift function with its parameters.

\section{Literature review}

The study of the properties of consistent and liquid oils is currently receiving much attention in the scientific literature on tribology. This is because lubrication is one of the most effective ways to improve machine durability. Lubrication is used in $99 \%$ of machine friction units. The article [2] analyzes the literature and provides an overview of the latest advances in modeling friction in lubricated contact. This review is based on a large body of data, identified gaps in the relevant area that can be used for future research. Greases represent an important group of lubricants due to their specific properties of adhesion to friction surfaces. One of the most common uses for greases is in rolling bearings for various applications. In work [3] the mechanisms of film formation during grease lubrication in final linear contacts were studied in order to improve the film-forming ability. For the research, an Elastohydrodynamic Lubrication (EHL) test bench was used with two interferometric microscopes that could simultaneously monitor two different contact points. The paper [4] studies the mechanisms of grease lubrication for rolling bearings. The evolution of a lubricating film during rotation of a glass disk in contact with an elastohydrodynamic lubrication (EHL) rolling is studied experimentally. The nature of the evolution of the fat film largely depended on the ranges of speeds and the structure of the lubricant. The main mechanisms that determine the formation of a film of grease in various lubricated contacts are revealed. In [5] considered are greases in wind turbine bearings to reduce wear. The research is completed by experiments with a contact model. Greases with a low base oil viscosity and high leak rate have been shown to be best suited, on average, for the tested conditions. In addition, the results of experiments with bearings are comparable to model experiments. Experimental studies of the tribological properties of greases: film thickness, coefficient of friction, and others are of great importance for practice. In [6] the physical properties and tribological properties of four greases were studied in detail, the worn surfaces were also investigated to understand the friction mechanism. The results show that calcium sulfonate complex grease (CSCG) has a high dropping point, good thermal and oxidative stability, excellent lubricating properties, which is mainly related to the nature and structure of the thickener and the synergism of the grease film and lubricant. In this article [7], lubricated contact at low sliding speeds is analyzed on a special tribometer under controlled boundary conditions. The coefficients of static and dynamic friction versus speed are obtained taking into account dry and lubricated contact. The influence of the coating on the surface of the DLC samples on the friction is also 
analyzed. The results demonstrated a significant effect of the relative sliding speed on the lubrication friction mechanisms. In [8] the thickness properties were investigated, according to which all greases form thicker than its counterpart liquid oil at low speeds. This suggests that the viscosity of the base oil determines the thickness of the lubricating film at low sliding speeds. Grease friction results indicate that the shape of the grease friction curves can differ significantly from the shape of the friction curves of liquid oils. Research on modeling work processes and mechanisms of grease lubrication of friction units is also of interest. The aim of this work [9] is to study the distribution of contact stresses and deformations of lubricated rolling bearings at high contact pressure. Threedimensional modeling by the finite element method using the elastoplasticity of the material is carried out and a comparison of the results with the classical law of elasticity is presented. The influence of lubrication, coefficient of friction, radial load has been investigated. The numerical results were compared with lubricated tribological tests under the same loading conditions. The presented results contribute to the tribological interpretation of the lubricated bearing degradation scenario without considering the mechanical properties of the lubricant. The aim of this article [10] is to simulate a mixed elastohydrodynamic lubrication (EHL) in real contact based on a grease rheological model. The model is designed to calculate the thickness of the lubricant film applied to a smooth and rough surface. The influence of load, surface roughness and rheological parameters on the thickness of the lubricating film was also investigated. It is concluded that the friction behavior of the lubricant depends on the thickness of the lubricating film, calculated according to the proposed EHL model.

At the same time, the analysis of literary sources shows that there are practically no mathematical descriptions of the mechanisms of grease lubrication, which is necessary for the creation of engineering methods for predicting and calculating the wear of machines.

\section{Theoretical principles of the method}

Contact interaction in a system consisting of two discs separated by a thin layer of grease is considered. The driving disc rotates at an angle $\varphi_{1}$ and through a layer of lubricant transfers the rotation to the second disc, which rotates at an angle $\varphi_{2}$. The difference between the angles of rotation $\varphi=\varphi_{1}-\varphi_{2}$ is equal to the relative angle of rotation. We assume that the dependence of the friction moment on the disk on the angle of relative rotation in the form of a power-law approximation is known from the experiment:

$$
M=c_{\varphi} \varphi^{n}
$$

where $c, n$ are the parameters of the function, which can be determined from the experimental points by the least squares method.

Parameters $c$ and $n$ can be approximately determined from two experimental points $\left(M_{1}, \varphi_{1}\right) ;\left(M_{2}, \varphi_{2}\right)$ :

$$
n=\frac{\lg \left(M_{1} / M_{2}\right)}{\lg \left(\varphi_{1} / \varphi_{2}\right)} ; c=\frac{M}{\varphi_{1}^{n}} .
$$

The second main relation in the formulation of the problem is the condition of equilibrium of the lubricant layer:

$$
M=\int_{F} \tau(r) r d F
$$

where $\tau(r)$ is the dependence of the relative shear forces (shear forces) on the radial coordinate $r ; F$ is the circular contact area. 
We will assume that between the tangential forces $\tau$ and the magnitude of the shear deformation $\gamma$ there is a power dependence of the form:

$$
\tau=G_{0} \gamma^{\alpha},
$$

where $\alpha, G_{0}$ is the dependency parameters.

The task is to determine the parameters $G_{0}$ and $\alpha$ according to the results of the test. Dependence of the magnitude of the layer shear $\gamma$ deformation on the rotation angle $\varphi$. By definition, the shear strain is equal to the ratio of the circumferential shear displacement to the dimension of the element along the axis $z$ (Fig. 1).

$$
\gamma=\frac{r d \varphi}{d z}=r \Theta
$$

where $\Theta$ is the relative twist angle; $r$ is the the radial coordinate of the layer point; $r d \varphi=V_{\varphi}$ is the relative circumferential shear displacement.

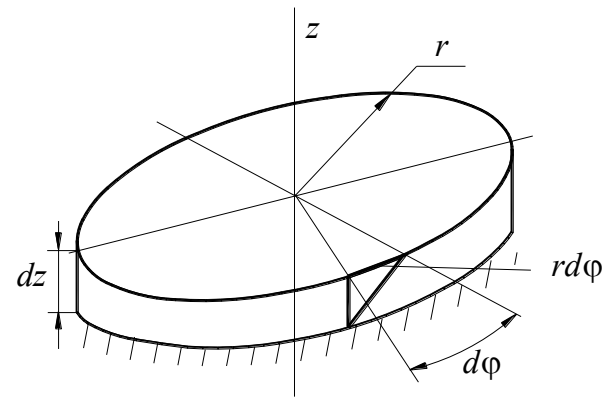

Fig. 1. Lubricant layer model

For a small layer thickness, you can take:

$$
\gamma=\frac{V_{\varphi}}{h}
$$

where $h$ is the grease layer thickness.

Equilibrium equation (3) for a circular layer with a diameter $d$ is written in the form:

$$
M=\int_{0}^{2 \pi} \int_{0}^{\frac{d}{2}} \tau r^{2} d \varphi=2 \pi \int_{0}^{\frac{d}{2}} \tau r^{2} d r .
$$

Taking into account (5), the maximum shear deformation:

$$
\gamma_{\max }=\frac{d}{2} \Theta \text {. }
$$

Taking the ratio (5) to (8), we have:

$$
r=\frac{d}{2} \frac{\gamma}{\gamma_{\max }}
$$

or in differential form:

$$
d r=\frac{d}{2 \gamma_{\max }} d \gamma
$$

Substituting (10) into (7), we have:

$$
M=\frac{\pi d^{3}}{4 \gamma_{\max }^{3}} \int_{0}^{\gamma_{\max }} \tau \gamma^{2} d \gamma .
$$


Experimental dependence (1) taking into account (5) at $r=\frac{d}{2}$ :

$$
\gamma_{\max }=\frac{d}{2} \Theta=\frac{\varphi}{2} \cdot \frac{d}{h}
$$

takes the form:

$$
M=c_{\varphi} \varphi^{n}=c_{\varphi}\left(\frac{2 h \gamma_{\max }}{\alpha}\right)^{n}
$$

or

where

$$
M=c_{\gamma} \gamma_{\max }^{n}
$$

$$
c_{\gamma}=c_{\varphi}\left(\frac{2 h}{d}\right)^{n} .
$$

Substituting (14) into (11), we obtain the equilibrium condition for the layer in the following form:

$$
c_{\gamma} \gamma_{\max }^{n}=\frac{\pi d^{3}}{4 \gamma_{\max }^{3}} \int_{0}^{\gamma_{\max }} \tau(\gamma) \gamma^{2} d \gamma .
$$

The solution of the problem in general form is represented as a series with any number of terms and is reduced to a system of algebraic equations. In this case, it makes sense to look for a solution in the form of a power function of the form:

$$
\tau(\gamma)=G_{0} \gamma^{\alpha}
$$

Substituting this function into (16), we have:

$$
c \gamma_{\max }^{n}=\frac{G_{0} \pi d^{3}}{4 \gamma_{\max }^{3}} \int_{0}^{\gamma_{\max }} \gamma^{\alpha+2} d \gamma
$$

or after integration:

$$
c_{\gamma} \gamma_{\max }^{n}=G_{0} \frac{\pi d^{3}}{4(\alpha+3)} \gamma_{\max }^{\alpha} .
$$

One resolving equation (19) contains two unknown quantities $G_{0}$ and $\alpha$. In this case, both unknowns can be found from one equation. From the condition of satisfying the equation for any values $\gamma_{\max }$ it follows:

and further, taking into account (14):

$$
n=\alpha
$$

$$
G_{0}=\frac{4 c_{\varphi}(n+3)}{\pi d^{3}}\left(\frac{2 h}{d}\right)^{n} .
$$

\section{Experimental Methodology}

To implement the test method, a device was used to determine the properties of plastic and other lubricants under conditions of a loaded friction lubricated contact. The design of the device is shown in Fig. 2. The device consists of a base on which an electric motor is mounted. The torque from the electric motor to the glass is transmitted through the gearbox 2. Disk 3 is rigidly fixed in the glass with a sample of lubricant 4 . A metal disk contacts the sample of lubricant 45 . The mass of the disk and the rod with the parts attached to them, as well as the mass of removable weights 6 , provides the creation of an axial load lubrication 
from the side of the measuring disk 5. The presence of the hinge provides an even distribution of the axial load over the entire contact surface of the disk. A lever is attached to the rod 7, which is in contact with the dynamometer 10 . The contact cone of the indicator 9 with the screw 8 rests against the upper end of the rod to measure the thickness of the lubricant layer. The shear stress is measured as the lubricant rotates over a metal steel disk pressed against it. The frictional force is recorded with a dynamometer. The axial load of the metal disc on the grease layer can be controlled by interchangeable steel weights. The area of the metal friction disk is $15 \mathrm{~cm}^{2}$. To test the interaction of a lubricant with a solid surface, the disc can be made of various materials with a given degree of roughness.

The shear stress of the disk $(\mathrm{Pa})$ for the lubricant at a given normal load is calculated by the formula:

$$
\tau=5 \cdot 10^{4} \cdot r \cdot F, \text { Па, }
$$

where $F$ is the force measured by dynamometer, $\mathrm{N} ; r$ is the lever arm, $\mathrm{m}(r=0,0475$ $\mathrm{m})$.

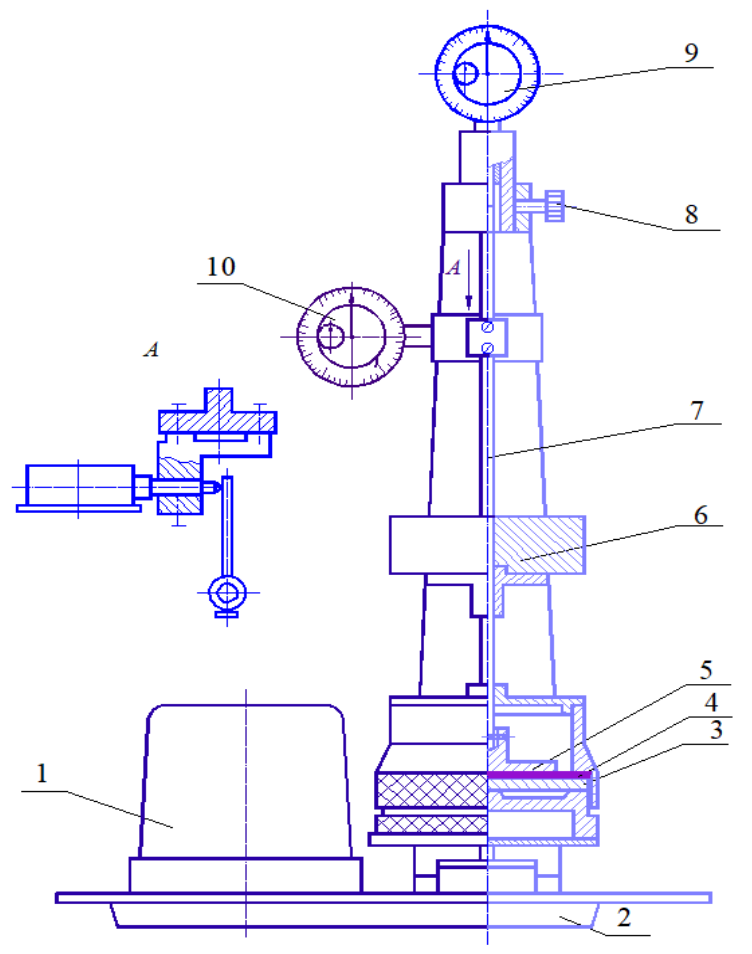

Fig. 2. Testing device

The device can obtain data to find the coefficient of friction $\mu$ and adhesive shear stress $\tau_{a}$ in accordance with the formula:

$$
\tau=\mu \sigma+\tau_{a}
$$

where $\sigma$ is the normal load on grease, $\mathrm{Pa}$.

A different number of weights allows you to get several experimental points, which, according to the expression, are plotted on a graph with coordinates $\tau$ and $\sigma$, then by a known method find $\mu$ and $\tau_{a}$. 


\section{Results and Discussion}

The tests were carried out on the device described above with the following initial data: $d=$ $43.7 \mathrm{~mm}$; normal load $\mathrm{Q}=25 \mathrm{~N}$; lower disc speed $0.077 \mathrm{rpm}$; lever arm on the indicator $l=$ $47.5 \mathrm{~mm}$; graduation price of the indicator: $1 \mathrm{~mm}-1.5 \mathrm{~N}$; Fiol-3 grease.

After turning on the drive, rotation from the lower disk through the layer of lubricant was transferred to the upper disk until the beginning of full slip, when the upper disk stopped. The time point was observed from the beginning of rotation to complete slippage with fixation every $10 \mathrm{~s}$ of the readings of the force indicator (movement of the lever of the force-measuring device). The results of measurements and their processing are presented in table. 1 .

Table 1. Results of measurements and calculations of shear properties of Fiol-3.

\begin{tabular}{|c|c|c|c|c|c|c|}
\hline Time, $t, \mathrm{~s}$ & 10 & 20 & 30 & 40 & 50 & 60 \\
\hline $\begin{array}{c}\text { Indicator readings, } \\
x, \mathrm{~mm}\end{array}$ & 2.3 & 4.2 & 5.2 & 5.5 & 5.7 & 6.0 \\
\hline $\begin{array}{c}\text { Lower disc swing angle, } \varphi_{2}, \mathrm{rad} \\
\text { Upper disc rotation angle, } \varphi_{2}, \mathrm{rad}\end{array}$ & 0.08 & 0.16 & 0.24 & 0.32 & 0.4 & 0.48 \\
\hline Displacement in the lubricant layer, $\varphi, \mathrm{rad}$ & 0.032 & 0.088 & 0.109 & 0.115 & 0.119 & 0.126 \\
\hline Frictional moment, $M, \mathrm{~N} \cdot \mathrm{mm}$ & 16.4 & 29.9 & 37.05 & 39.18 & 40.6 & 42.7 \\
\hline
\end{tabular}

A graphic interpretation of the dependence of the friction moment in the lubricant layer on the relative angular displacement is shown in Fig. 3.

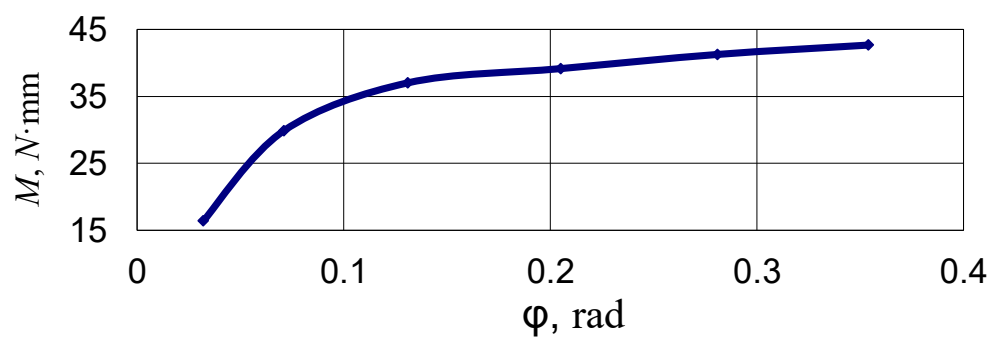

Fig. 3. Dependence of the friction moment on the angle of displacement of the grease layer.

The parameters of dependence (1) can be found from experimental data using the least squares method or using the Excel program. Further, the parameters of the oil film shift function (4) are determined by the formulas (19-21). Determination of the parameters of the power-law approximation of the function. In accordance with (1), we will perform calculations according to (2). Let's take a look at the table. 1 by two points:

$$
M_{1}=16.4, \mathrm{H} \cdot \text { мм; } \varphi_{1}=0.032 \text { рад; } M_{2}=42.7, \mathrm{H} \cdot \text { мм; } \varphi_{2}=0.354 \mathrm{rad} \text {. }
$$

По формуле (2):

$$
n=\frac{\lg (16.4 / 42.7)}{\lg (0.032 / 0.354)}=0.3981 \cong 0.4 ; c_{\varphi}=\frac{16.4}{(0.032)^{0,3981}}=64.56 \mathrm{~N} \mathrm{~mm} /(\mathrm{rad})^{\Pi} .
$$

By formula (20), we find: $\alpha=n=0.4$, and according to the formula (21):

$$
G_{0}=\frac{4 \cdot 64.56(0.4+3)}{\pi \cdot 43.7^{3}}\left(\frac{2 \cdot 0.001}{43.7}\right)^{0.4}=61.57 \mathrm{~Pa} .
$$


The thickness of the film is taken to be $1 \mu \mathrm{m}$; in order to increase the accuracy of calculations, it must be determined experimentally. Thus, the shear diagrams of a thin layer from Fiol-3 grease are described by the dependence:

$$
\tau=61.57 \gamma^{0,4} \mathrm{~Pa} \text {. }
$$

In appearance, the curve of this function is similar to the curve in Fig. 3, since the exponents of these curves coincide $n=\alpha$.

\section{Conclusions}

1. When studying the wear of surfaces under boundary lubrication conditions, it is necessary to know the stiffness characteristics of a thin layer of lubricant under normal and tangential stresses. Previous studies have examined the bulk mechanical properties of lubricants.

2. A method for determining the tangential stiffness properties of thin films is proposed. The method is based on twisting a round thin layer of grease between two rigid circular discs.

3. The contact mechanics of torsion of a thin circular lubricant layer has been developed and relations for determining the parameters of the shear diagram of a thin lubricant layer have been obtained.

4. A method for torsion testing of a thin lubricant layer with the determination of the parameters of the film shear diagram has been developed.

5. According to the developed method, the Fiol-3 grease was tested and the parameters of the shear diagram were determined.

\section{References}

1. A.V. Dykha., A.G. Kuzmenko, Journal of Friction and Wear, 2, 138 (2015)

2. Z.A. Khan, V. Chacko \& H. Nazir, Friction, 5, 1 (2017). https://doi.org/10.1007/s40544-017-0143-0

3. Z. Wang, X. Shen, X. Chen, X. et al., Friction, 7, 237 (2019). https://doi.org/10.1007/s40544-018-0208-8

4. X. Li, F. Guo, G. Poll, et al., Friction, 9, 179 (2021)

5. F. Schwack, N. Bader, J. Leckner, C. Demaille, G. Poll, Wear, V. 454-455, 203335 (2020)

6. X. Fan, W. Li, H. Li, M. Zhu, Y. Xia, J. Wang, Tribology International, 118, 128 (2018)

7. I. Ghezzi, D. Tonazzi, M. Rovere, C. Le Coeur, Y. Berthier, F. Massi, Tribology International, Volume 155, 106788 (2021)

8. B. Vengudusamy, C. Enekes, R. Spallek, Tribology International, 129, 323 (2019)

9. D. Tonazzi, E. Houara Komba, F. Massi, G. Le Jeune, J.B. Coudert, Y. Maheo, Y. Berthier, Wear, 376 B, 1164 (2017)

10. D. Wang, J. Yang, P. Wei, W. Pu, Tribology International, 154, 106710 (2021) 